\title{
2D scanning Rotman lens structure for smart collision avoidance sensors
}

\author{
Leonard T. Hall ${ }^{a, b}$, Hedley J. Hansen ${ }^{a, b, c}$, and Derek Abbott ${ }^{a, b}$ \\ ${ }^{a}$ Centre for Biomedical Engineering The University of Adelaide, SA 5005 Australia \\ ${ }^{b}$ Department of Electrical and Electronic Engineering, The University of Adelaide, SA 5005 \\ Australia \\ ${ }^{c}$ Electronic Warfare \& Radar Division, DSTO, Edinburgh, SA 5111 Australia
}

\begin{abstract}
Although electronically scanned antenna arrays can provide effective mm-wave search radar sensors, their high cost and complexity are leading to the consideration of alternative beam-forming arrangements. Rotman lenses offer a compact, rugged, reliable, alternative solution. This paper considers the design of a microstrip based Rotman lens for high-resolution, frequency-controlled scanning applications. Its implementation in microstrip is attractive because this technology is low-cost, conformal, and lightweight. A sensor designed for operation at 77 $\mathrm{GHz}$ is presented and an $\sim 80^{\circ}$ azimuthal scan over a $30 \mathrm{GHz}$ bandwidth is demonstrated.
\end{abstract}

Keywords: Rotman lens, Microstrip, Frequency scanned array, Antenna array, Collision avoidance

\section{INTRODUCTION}

Electronically scanned antenna arrays have been used effectively to scan in one or two dimensions. Traditionally, their cost and complexity make them unsuitable for most applications. For these reasons much work has been carried out to find a low-cost and simple beam steering arrangement. Frequency scanning makes use of the variation of wavelength to implement a phase change across a linear antenna array. A simple example is the series fed patch antenna array. Constrained lenses have also been used quite successfully to scan in one dimension. The most promising of these arrangements is the Rotman lens.

This paper is concerned with the design and development of constrained lens solutions that provide an alternative to electronically scanned systems. A Rotman lens, built using microstrip techniques feeding a frequency scanned patch antenna array, provides high gain, large scan angles, conformal geometry, and low cost. The Rotman lens makes available many narrow staring beams in the horizontal plane, which may be scanned in the vertical plane by varying the frequency of operation. Scan angles of $60^{\circ}$ in both planes and high gains are obtainable with careful design.

\section{ROTMAN LENS}

A Rotman lens consists of a parallel plate region (body) with beam ports and array ports distributed along opposite contours. The central beam port provides equal path lengths to each array element. An offset beam port produces a path length difference and hence a phase gradient along an array, giving a steered beam.

The design of the lens is governed by the Rotman-Turner design equations ${ }^{1}$ that are based on the geometry of the lens; these are shown in Fig. 1. The equations generate the positions of the antenna ports based on three perfect focal points $\left(F_{0}, F_{1}\right.$, and $\left.F_{2}\right)$. The beam ports are placed on a circular arc joining the three focal points. The lower case letters represent their upper case variable normalised to the aperture size (maximum $N$ ), and $w$ is the phase delay in wavelengths between the antenna port and patch antenna. The defining parameters of the Rotman lens are the internal scan angle $\alpha$, ratio of $\frac{G}{F}$, value of $f$ ( $F$ normalised to the aperture size maximum $N)$ and the number of antenna ports (external scan angle).

Further author information: (Send correspondence to L. T. Hall)

L. T. Hall: E-mail: lthall@eleceng.adelaide.edu.au, Telephone: 0402336642

H. J. Hansen: E-mail: hedley.hansen@dsto.defence.gov.au

D. Abbott: E-mail: dabbott@eleceng.adelaide.edu.au 


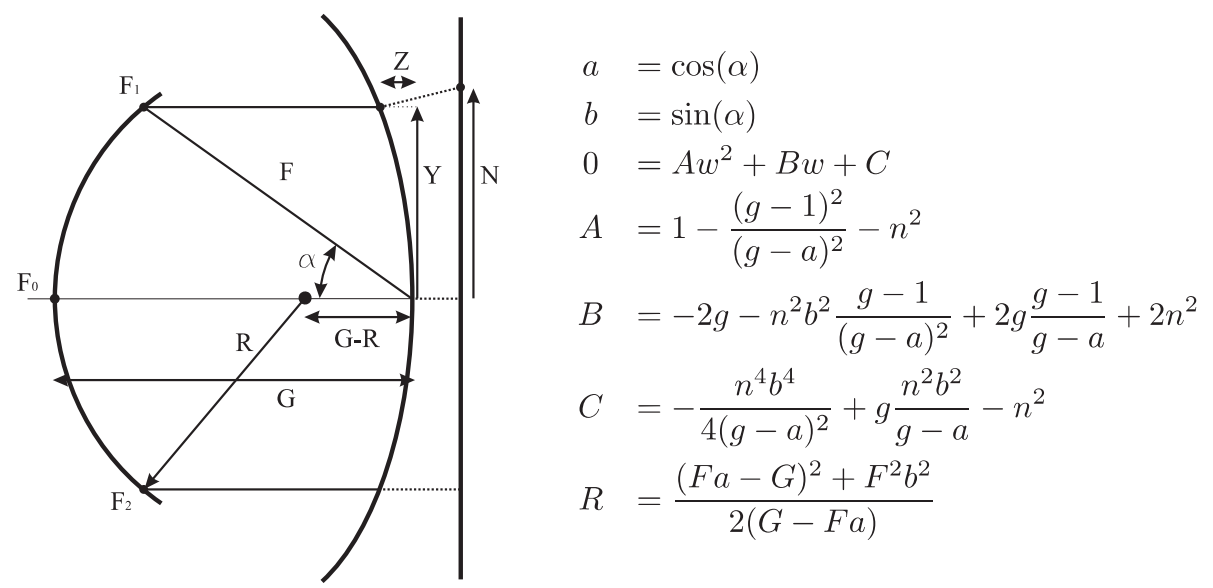

Fig. 1. Rotman lens topology ${ }^{1}$

\subsection{Rotman Lens Design, Rules of Thumb}

These rules of thumb have been used to quickly design cheap lenses for applications that do not require high levels of efficiency or gain. They tend to be applied to lenses with three to five beam ports with seven to fifteen antenna ports. These lenses can achieve approximately $10 \mathrm{~dB}$ sidelobes but they tend to suffer from poor isolation between ports and high sidelobes outside the scan angle due to reflections from the lens' sidewalls.

Typical rules of thumb are:

- $0.8 \leq f \leq 1$.

- Distance between ports ${ }^{1}$ and antennas of $\leq \frac{\lambda}{2}$.

- Taper ports ${ }^{2}$ with angle $\leq 12.5^{\circ}$.

- Sidewalls have absorptive material or matched dummy ports. ${ }^{2}$

- Antenna lines are bent to implement required phase delays. ${ }^{3-5}$

\subsection{High Performance Rotman Lens}

We now summarise the design procedure for delivering high performance Rotman lenses. ${ }^{6-10}$

The lack of an automation procedure, which overcomes the time consuming fine tuning simulation, has hindered the deployment of efficient Rotman lens sensors. Our solution is to use Matlab to generate the lens design and export it to Autocad's Direct Exchange Format (DXF). This prepares the design for simulation (using Ensemble) or manufacture.

Historically reflections from the sidewalls are the biggest limiting factor affecting performance. The sidewalls designed to minimise the reflected radiation within the lens. This involves evoking two sets of dummy ports, one, dealing with radiation from the antenna ports and the other dealing with the radiation from the beam ports. A typical orientation for well designed sidewalls is shown in Fig. 2. Little energy from the antenna ports is incident on the beam dummy ports and any energy that is present, is reflected directly onto the antenna dummy ports.

The S-parameter impedance predictions are efficiently calculated using Ensemble software.

Port design and matching have been addressed through iteratively matching each port to the body of the lens and then recalculating the S-matrix for the new port impedance. The fields around the port are shaped to approximate those in the parallel plate region of the body of the lens.

An excitation taper has been introduced to minimise sidelobes by varying $f$. Fig. 3 shows the variation in shape of a Rotman lens as we vary $f$ keeping the port spacing and aperture size constant. Fig. 3(c) shows 


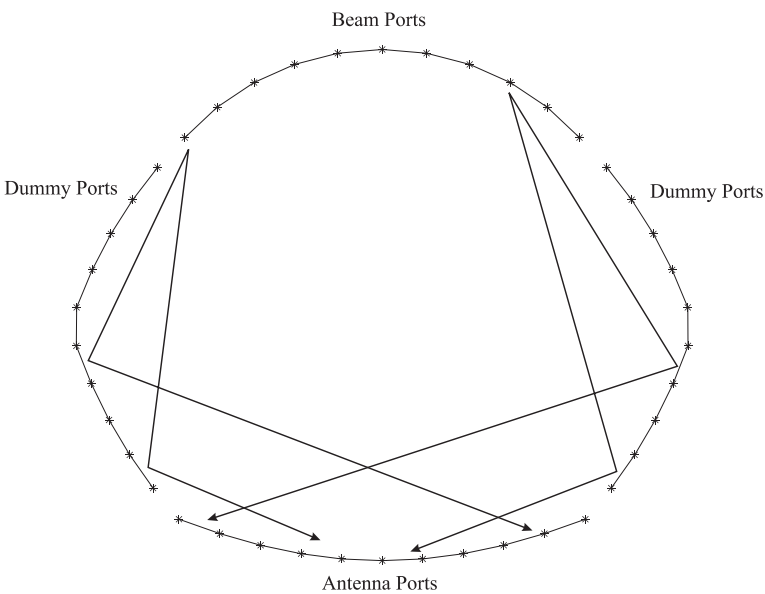

(a) Poorly designed sidewalls

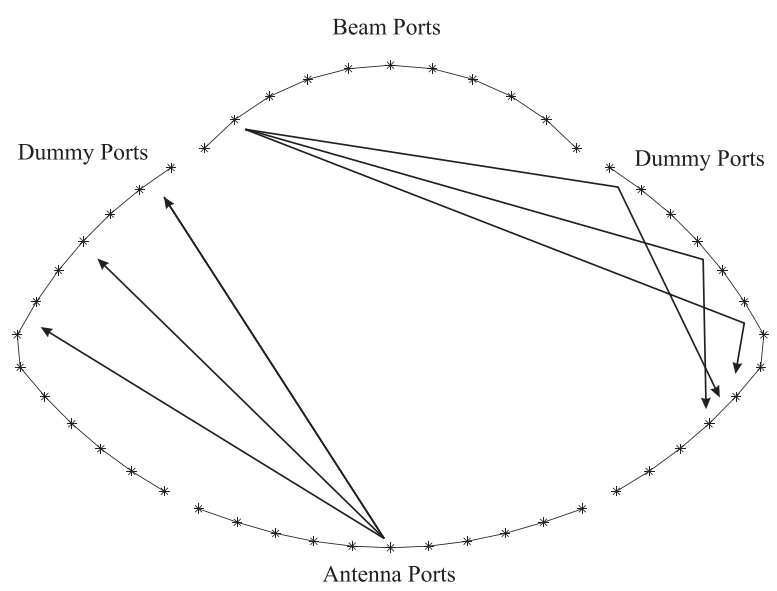

(b) Well designed sidewalls

Fig. 2. Sidewall design, demonstrating that energy not absorbed by the dummy ports need not be directed back onto the antenna or beam ports.

that keeping the half wavelength spacing between antenna ports problematic for $f \leq 0.75$ for the outer antenna ports. We have also maximised the beam-port widths for improving their isolation and for directing more energy towards the inner antenna ports. Increasing the beam port width beyond $\lambda / 2$ is not possible as unwanted higher order modes become excited, however, it is possible to excite two or more ports side by side to get an effective increase in port width. ${ }^{11}$ This is desirable because the effective increase in aperture size leads to a narrowing of the beam pattern, within the body of the lens, thus reducing energy lost to the sidewalls. By coupling two adjacent ports together a total gain of up to $3 \mathrm{~dB}$ is achieved and sidelobes are reduced by as much as $5 \mathrm{~dB}$ at the expense of slightly increased beam width.

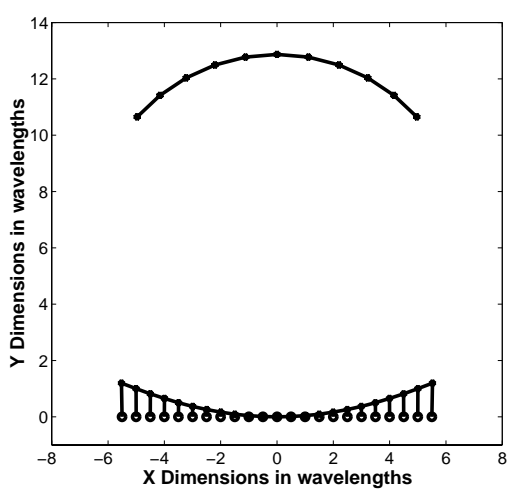

(a) $f=1$

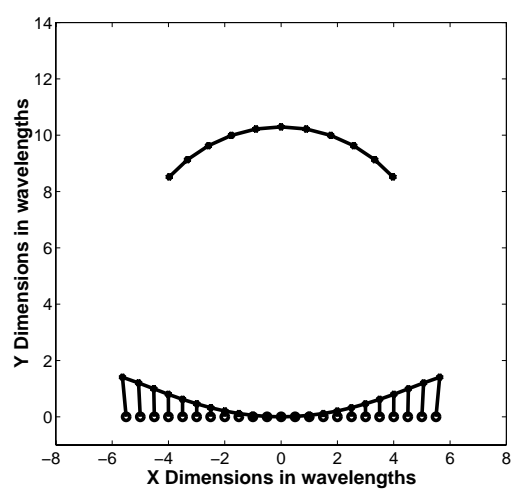

(b) $f=0.8$

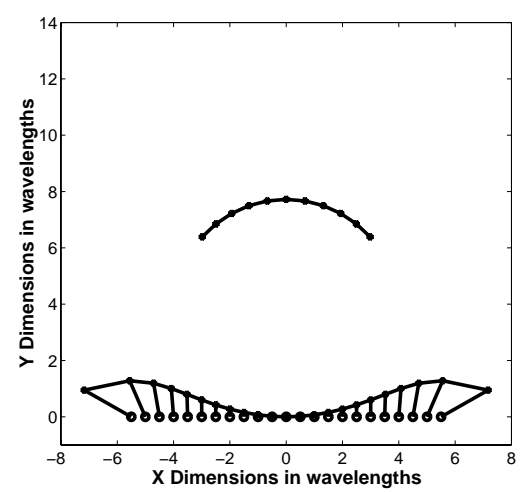

(c) $f=0.6$

Fig. 3. By changing the parameter $f$, the height of the Rotman lens (in the figures above), the shape of the antenna ports is changed, allowing us to implement crude excitation tapering. 


\subsection{Improving Bandwidth}

The absence of any frequency dependance in the Rotman equations, shown in Fig. 1, exhibits on absence in frequency dependance, suggesting that the Rotman lens configuration is inherently broad bandwidth. Nevertheless the following are key factors that limit bandwidth in any Rotman lens implementation.

\subsubsection{Impedance transformation}

Both the Beam and Antenna ports require matching to a standard transmission line. A quarter wavelength impedance transformer generally achieves this but this can be unsatisfactory when broad bandwidth is required. In such instances an exponential taper is pertinent. An exponential impedance taper is a half-wavelength line whose impedance varies in an exponential fashion between the load and source impedances. Fig. 4 shows that for normalised frequencies of greater than 1 the exponential impedance taper provides a much better match than the quarter wavelength transformer. The designs represented here achieve impedance transformation using a exponential impedance taper designed for the lowest desired frequency.

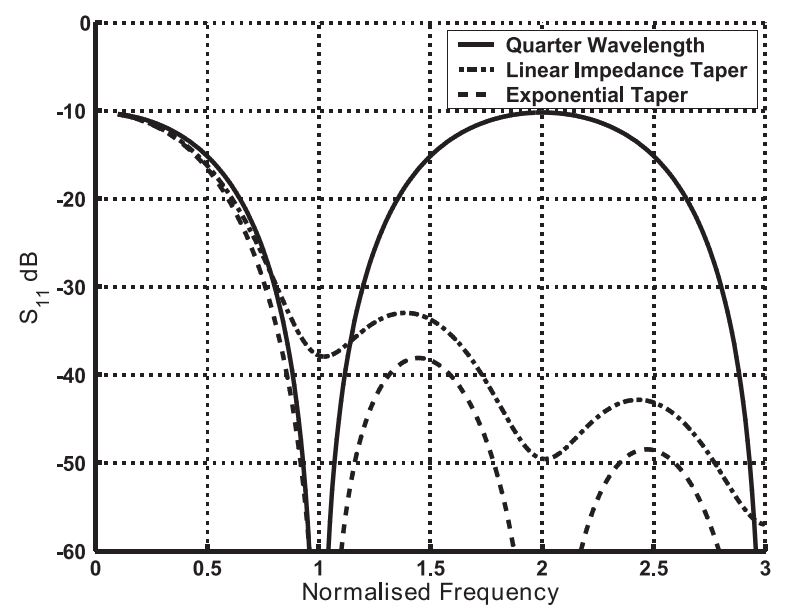

Fig. 4. Theoretical reflection coefficient $\left(S_{11}\right)$ of various impedance transformers from $25 \Omega$ to $100 \Omega$. The figure clearly shows the improvement in $\mathrm{S}_{11}$ when using an exponential tapered line instead of a quarter wavelength transformer. The frequency is normalised by dividing the frequency range by the design frequency.

\subsubsection{Dummy ports and sidewalls}

Reflection reduction, from the sidewalls of the Rotman lens, is critical for obtaining acceptable performance of the Rotman lens. The additional requirement of broad bandwidth further challenges the designer in this area. The dummy ports or absorptive media must provide an effective match across the entire bandwidth of the device.

\subsubsection{Port spacing}

The distance between adjacent ports is limited by the presence of sidelobes inside the body of the lens. Port spacing beyond half a wavelength cause the antenna ports to direct a portion of there energy towards the, sidewalls of the lens, antenna ports, or beam ports. This reduces efficiency, increases mutual coupling between beam ports, and increases sidelobe levels. Ports are designed for the highest operating frequency, and spaced less than half a wavelength apart.

\subsubsection{Aperture size}

Although the beam angle of any given beam port is independent of frequency, the beam width and antenna gain are not. As frequency increases the effective aperture increases in size, increasing gain and reducing beam width. This can lead to unacceptably narrow beams at high frequencies, and poor gain at low frequencies. Coupled beam ports can solve this problem by focusing energy onto the central antenna ports of the lens for high frequencies, while at low frequencies the entire all antenna ports would be excited. This would effectively reduce the aperture size for high frequencies. 


\section{FREQUENCY SCANNED ANTENNA}

One realisation of a frequency-scanned antenna is the series fed patch antenna array shown in Fig. 5 . The antenna structure is made up of 20 patch antennas each connected in series by short line segments. The radiation excitation of each patch antenna is varied by changing the width of each successive transition line segment according to an exponential taper

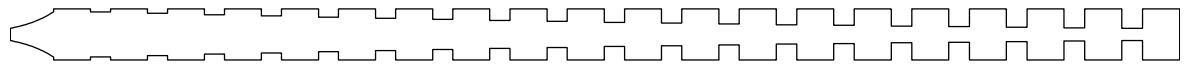

Fig. 5. Series fed patch antenna array made up of 20 patch elements with interconnecting lines tapering from $20 \Omega$ to $50 \Omega$. On the left is an exponential impedance taper to $50 \Omega$. This array is designed to have a beam angle of $30^{\circ}$ at $77 \mathrm{GHz}$

The limitation of this layout is that the array can only be matched over a large bandwidth for scan angles away from broadside (ie. normal from the ground plane). At broadside the patch elements are excited in phase and their impedances sum together, resulting in a very large input impedance. For scan angles between $15^{\circ}$ and $85^{\circ}$, a match can be achieved of better than $15 \mathrm{~dB}$ across the entire range in an antenna configuration comprising of 60 elements or more. The reflection coefficient for such a configuration designed for operation at $77 \mathrm{GHz}$ is shown in Fig. 6(a) and radiation patterns at selected frequencies in Fig. 6(b).

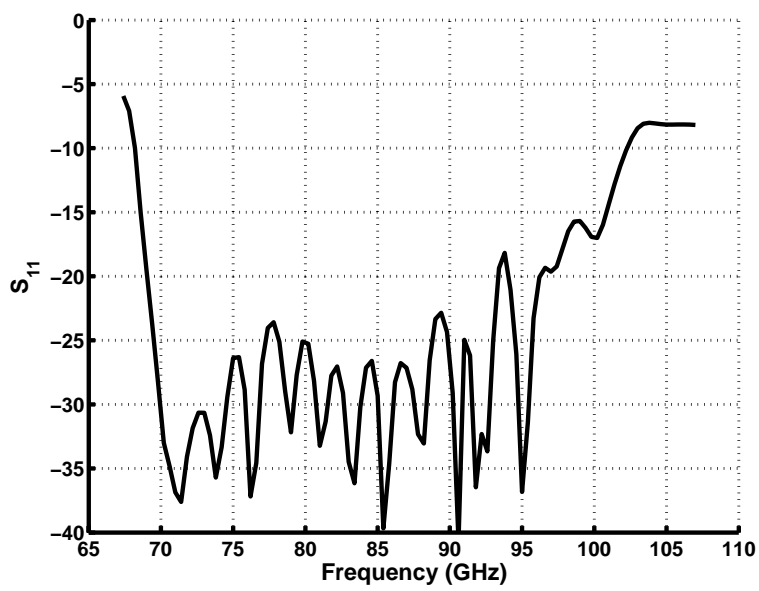

(a) Reflection coefficient

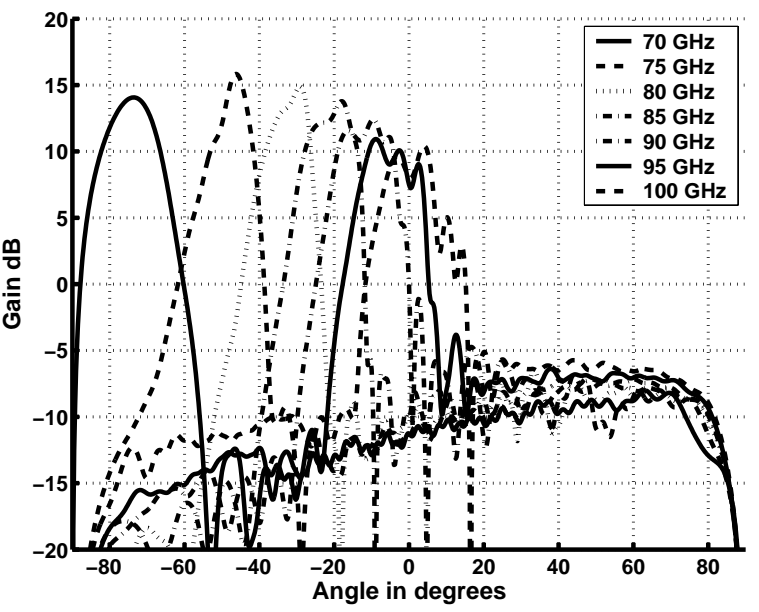

(b) Beam patterns

Fig. 6. Simulated performance of a series fed patch antenna array. $\epsilon_{r}=3.6$, substrate thickness $=0.127 \mathrm{~mm}$, patch size $0.94 \mathrm{~mm}$ by $1.3 \mathrm{~mm}$, connecting lines vary between $20 \Omega$ to $50 \Omega$ with an exponential dependance. These figures demonstrate the exceptional bandwidth and scan angles that may be achieved using this simple approach.

The transmitted beam angle can be calculated using the following formula,

$$
\cos \theta=\lambda\left(\frac{1}{d}-\frac{1}{\lambda_{g}}\right)
$$

where $\lambda$ is the free space wavelength, $\lambda_{g}$ is the average guided wavelength and $d$ is the pitch of the patch antenna array (ie. center-to-center distance between patches). By setting $\cos \theta$ to 1 or 0 , and $\lambda_{g}=k \lambda$ the formula can be rearranged to determine the maximum obtainable bandwidth. The maximum and minimum frequencies of the band are given by 


$$
\begin{gathered}
f_{\max }=\frac{k}{d} \\
f_{\min }=\frac{k}{d(1+k)} .
\end{gathered}
$$

These expressions show that as the substrate dielectric constant increases (smaller $k$ ) the bandwidth will become narrower, while maintaining the same scan angle, increasing the change in scan angle per unit frequency. The configuration suffers from the unusual situation of having to much bandwidth to make full use of the scanning potential of these antenna. Substrates with relative dielectric constant $>10$ achieve scan angles of $30^{\circ}$ with acceptably small bandwidth.

\section{PROPOSED IMPLEMENTATION}

We propose the Rotman lens design shown in Fig. 7 for both transmit and receive sensing applications

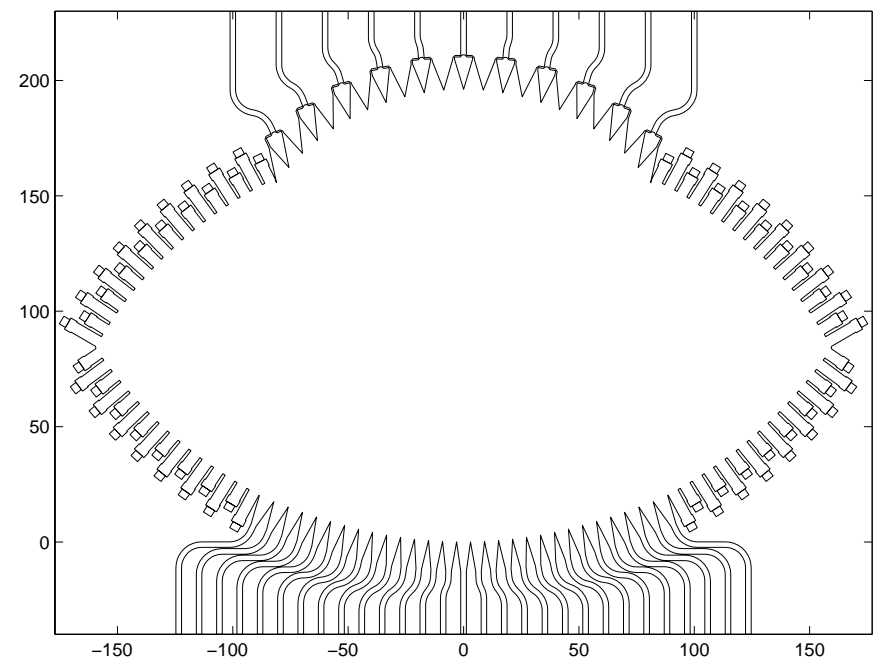

(a) Rotman Lens

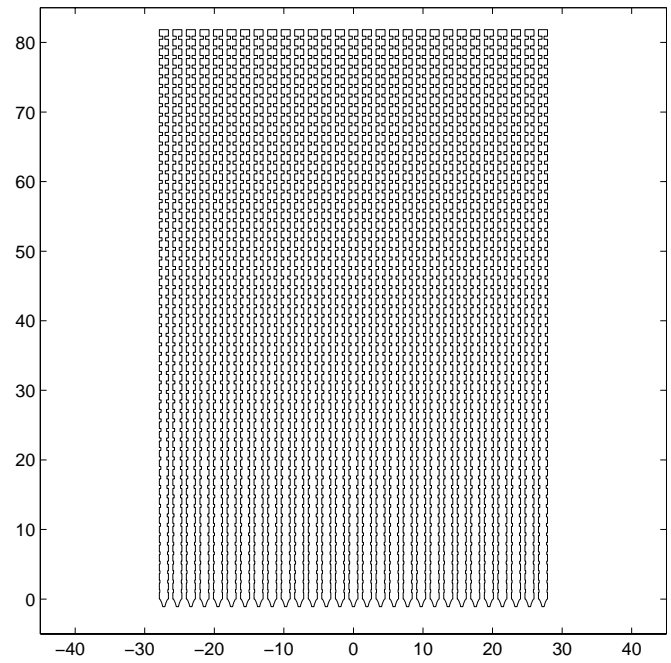

(b) Antenna Array

Fig. 7. The geometry shown above is printed on either side of a double layered substrate, separated by a ground plane. The Rotman lens is printed underneath while the antenna array on the right is printed on top allowing it to be mounted flush with the exterior of the vehicles body. Coupling between the upper and lower layers is achieved by slots in the ground plane between two microstrip lines.

The lens design shown in Fig. 7(a) has 29 antenna ports and 11 beam ports each made up of two subports. The frequency steered patch antenna arrays in Fig. 7(b) are fed by slot in the ground plane under the microstrip line at the base of the antenna. Ongoing work is being carried out to fabricate and test the design - target performance parameters are shown in Table 1.

\section{CONCLUSION}

This paper demonstrates the feasibility of a lens arrangement, consisting of a Rotman lens back end driving an array of frequency scanned antenna, capable of scanning in two dimensions. The final performance of this arrangement will be limited by the maximum achievable bandwidth of the Rotman lens. For collision avoidance applications a bandwidth of $5 \mathrm{GHz}$ would make possible a system with horizontal scan angles of $60^{\circ}$ and vertical scan angles of $25^{\circ}$, greatly enhancing the capability of the sensor. 
Table 1. Target Lens Performance

\begin{tabular}{|l|l|}
\hline Bandwidth & $>5 \mathrm{GHz}$ \\
\hline Gain of Central Beam & $>25 \mathrm{~dB}$ \\
\hline Horizontal Beam Width & $4^{\circ}$ \\
\hline Vertical Beam Width & $10^{\circ}$ \\
\hline Horizontal Beam Crossover & $-7 \mathrm{~dB}$ \\
\hline Vertical Beam Crossover & $-5 \mathrm{~dB}$ \\
\hline Sidelobe & $<-15 \mathrm{~dB}$ \\
\hline Total Insertion loss & $<10 \mathrm{~dB}$ \\
\hline
\end{tabular}

\section{ACKNOWLEDGMENTS}

Funding from the DSTO RF Hub and the Sir Ross and Sir Keith Smith fund is gratefully acknowledged.

\section{REFERENCES}

1. W. Rotman and R. F. Turner, "Wide angle microwave lens for line source applications," IEEE Trans. Antennas Propag. AP-11, pp. 623-632, November 1963.

2. L. Musa and M. S. Smith, "Microstrip port design and sidewall absorption for printed Rotman lenses," Proc. IEE Proceedings Microwaves, Antennas and Propagation 136, pp. 53-58, February 1989.

3. D. Abbott and A. Parfitt, "Collision avoidance device using passive millimetre-wave array based on insect vision," in Proc. IREE 14th Australian Microelectronics Conference (Micro' 97), pp. 201-204, (Melbourne), October 1997.

4. D. Abbott and A. J. Parfitt, "Extension of the insect-vision paradigm to millimeter waves," in Proc. SPIE Transportation Sensors, 3207, pp. 103-106, (Pittsburg), October 1998.

5. A. Mohamed, A. Campbell, D. Goodfellow, D. Abbott, H. Hansen, and K. Harvey, "Integrated millimetre wave antenna for early warning detection," in Proc. SPIE Design, Characterization and Packaging for MEMS and Microelectronics, 3893, pp. 461-469, (Queensland), October 1999.

6. L. Hall, D. Abbott, and H. Hansen, "Design and simulation of a high efficiency Rotman lens for mm-wave sensing applications," in Proc. 2000 Asia Pacific Microwave Conference, pp. 1419-1422, (Sydney), December 2000.

7. L. Hall, D. Abbott, and H. Hansen, "Design of smart multi-beam mm-wave antennas," Proc. SPIE 2000 Smart Electronics and MEMS II 4236, pp. 188-193, December 2000.

8. L. Hall, H. Hansen, and D. Abbott, "Design and simulation of a high efficiency Rotman lens for mm-wave collision avoidance sensor," Microelectronics Journal, December 2001.

9. L. Hall, D. Abbott, and H. Hansen, "Microstrip-based Rotman lens for mm-wave sensing operations," Proc. SPIE Passive Millimeter Wave Technology V 4373, pp. 40-48, (Orlando), April 2001.

10. L. Hall, D. Abbott, and H. Hansen, "Monolithic fabrication of Rotman lenses," in Proc. SPIE Design Charactization and Packaging for MEMS and Microelectronics II, 4593, pp. 119-127, (Adelaide), December 2001.

11. A. K. S. Fong and M. S. Smith, "A microstrip multiple beam forming lens," Radio and Elec. Eng 54, pp. 318-320, 1984. 